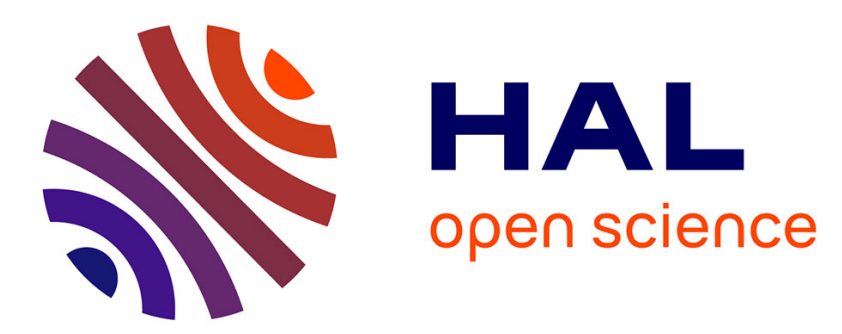

\title{
Utilisation des anticoagulants chez les patients âgés atteints de fibrillation atriale dans un service de cardiogériatrie
}

H. Yang, W. Jarzebowski, C. Nouhaud, J. Belmin, Carmelo Lafuente-Lafuente

\section{- To cite this version:}

H. Yang, W. Jarzebowski, C. Nouhaud, J. Belmin, Carmelo Lafuente-Lafuente. Utilisation des anticoagulants chez les patients âgés atteints de fibrillation atriale dans un service de cardiogériatrie. Annales de Cardiologie et d'Angéiologie, 2016, 10.1016/j.ancard.2016.04.025 . hal-01323777

\section{HAL Id: hal-01323777 \\ https: / hal.sorbonne-universite.fr/hal-01323777}

Submitted on 31 May 2016

HAL is a multi-disciplinary open access archive for the deposit and dissemination of scientific research documents, whether they are published or not. The documents may come from teaching and research institutions in France or abroad, or from public or private research centers.
L'archive ouverte pluridisciplinaire HAL, est destinée au dépôt et à la diffusion de documents scientifiques de niveau recherche, publiés ou non, émanant des établissements d'enseignement et de recherche français ou étrangers, des laboratoires publics ou privés. 


\section{Utilisation des Anticoagulants chez les Patients Âgés Atteints de Fibrillation Atriale dans un Service de Cardiogériatrie}

Anticoagulation use in older patients with atrial fibrillation in a cardio-geriatric unit

\section{AUTEURS :}

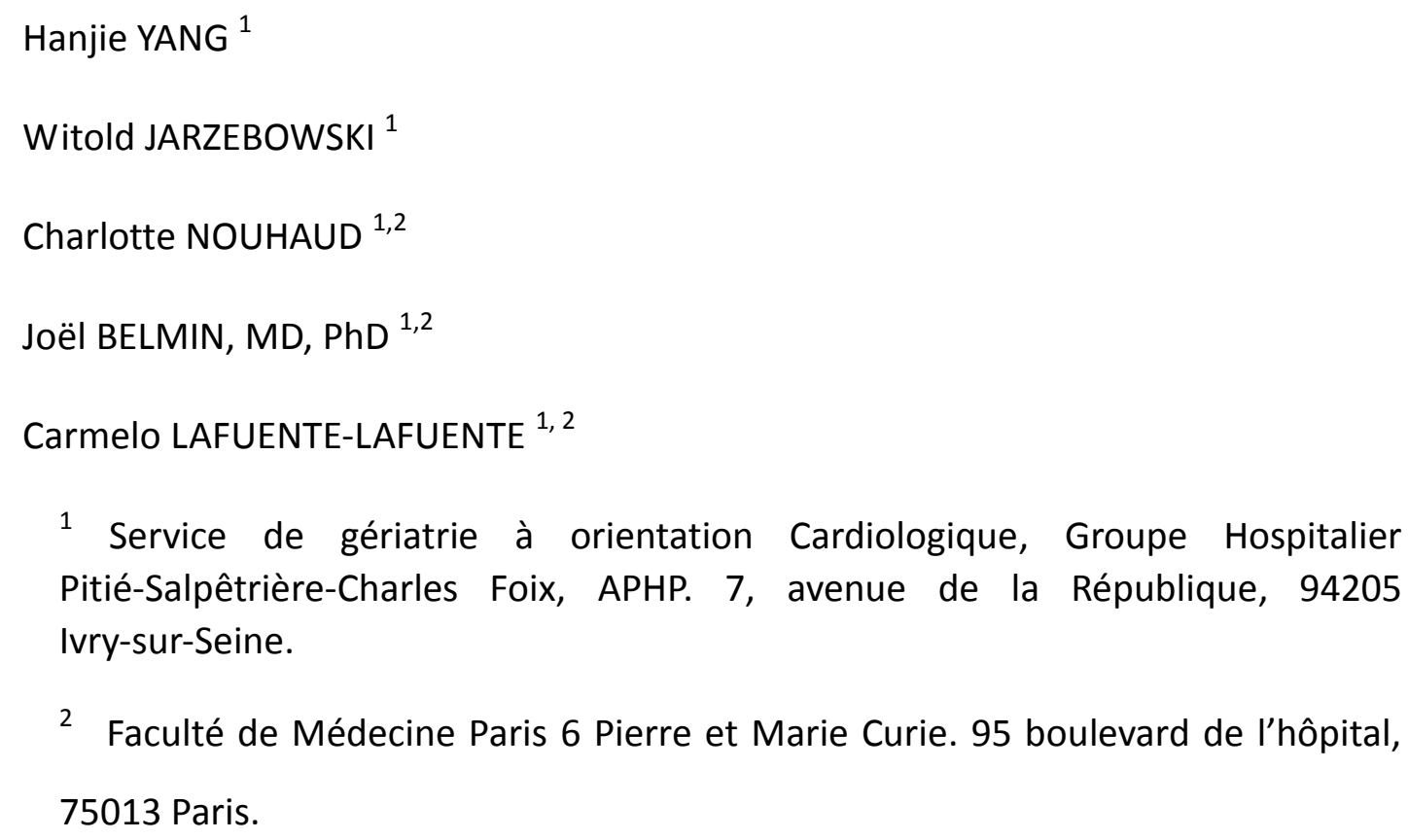

CORRESPONDANCE À :

Dr. Carmelo LAFUENTE-LAFUENTE

Service de gériatrie à orientation Cardiologique, Hôpital Charles Foix

DHU FAST, Faculté de Médecine Paris 6 Pierre et Marie Curie

7, avenue de la République. 94205 Ivry-sur-Seine.

Tel : +33149594356/4565 Fax : +33149594383

E-mail: carmelo.lafuente@cfx.aphp.fr

E-mail 2: c.lafuente@nodo3.net 
NomBRE DE MOTS : Résumé : 250 ; Texte : 2320.

Tableaux: 4 ; Figures: 0 ; Références: 16.

SOURCES DE FINANCEMENT : Aucune.

CONFLITS D'INTERET : HY : aucun, WJ : aucun, CN : aucun, JB : aucun, CLF : aucun. 


\section{Résumé}

But de l'étude : Les patients âgés atteints de fibrillation atriale (FA) ont un risque embolique élevé mais ils sont moins souvent anticoagulés. Nous voulions évaluer nos pratiques dans un service de gériatrie spécialisé.

Patients et Méthode : étude observationnelle descriptive incluant tous les patients de plus de 75 ans avec une FA hospitalisés pendant une période de 3 mois dans un service de cardio-gériatrie. Leur risque embolique (score CHADS2), risque hémorragique (score HAS-BLED), traitement anti-thrombotique à l'arrivée et toute modification postérieure, ont été recueillis.

Résultats : 34 patients ont été inclus (âge moyen : 85 ans). La FA était déjà connue chez 28 patients, dont 20 suivaient à l'arrivée une anticoagulation efficace (10 fluindione, 9 warfarine, 1 dabigatran), 4 un traitement par aspirine et 4 aucun traitement anti-thrombotique. Seul le traitement d'un de ces patients a été modifié, en remplaçant l'aspirine par warfarine. La FA a été une découverte à l'occasion de leur hospitalisation chez 6 patients, dont 4 ont été mis sous anticoagulation (3 warfarine, 1 fluindione).

Au total, 9 patients (26\%) sont sortis de l'hôpital sans une anticoagulation. Les raisons invoquées étaient : démence évoluée (4 patients), risque hémorragique jugé excessif (4), risque embolique jugé faible (1). Il y a eu une nette tendance à d'avantage d'initiation de l'anticoagulation chez les patients avec une FA nouvellement découverte $(p=0,09)$.

Conclusions: Un nombre non négligeable de patients âgés avec FA restent sans traitement anticoagulant même après passage dans un service spécialisé. La démence évoluée et le risque hémorragique sont les raisons les plus fréquemment invoquées.

Mots-clés : fibrillation atriale, patient âgé, anticoagulants, embolie, risque hémorragique 


\section{Abstract}

Objective: Older people with atrial fibrillation (AF) have an increased embolic risk but they are less frequently treated with anticoagulants. We wanted to assess our current practice in a specialized service.

Patients and Methods: prospective observational study including all patients older than 75 years admitted during 3 months in a cardio-geriatric unit. Patients' embolic risk (CHADS2 score), hemorrhagic risk (HAS-BLED score), anti-thrombotic treatment at arrival and any modification afterwards, were analyzed.

Results: 34 patients were included (mean age: 85 years). AF was known in 28 patients, of whom 20 were under anticoagulant therapy at their admission (10 fluindione, 9 warfarine, 1 dabigatran), 4 received aspirin and 4 no antithrombotic treatment. Only the treatment of one of these patients was modified, replacing aspirin by warfarin. AF was newly diagnosed in 6 patients, of whom anticoagulation were initiated in 4 patients ( 3 warfarine, 1 fluindione).

Finally, 9 patients (26\%) left the hospital without anticoagulant treatment. Reasons given by their attending doctors were: advanced dementia (4 patients), estimated excessive hemorrhagic risk (4), and estimated low embolic risk (1). There was a clear trend to initiate anticoagulation more frequently in patients with a newly diagnosed $\operatorname{AF}(p=0.09)$

Conclusions: a substantial proportion of older patients with AF do not receive anticoagulant therapy, even after been admitted to a specialized service. Advanced dementia and hemorrhagic risk are the reasons most frequently given for that.

Keywords: atrial fibrillation, older patients, anticoagulants, embolism, hemorrhagic risk 


\section{Introduction}

La fibrillation atriale (FA) est la plus fréquente des arythmies soutenues en pratique clinique. Elle a une association très forte avec l'âge. La majorité des cas de FA se produisent chez les patients de plus de 60-70 ans et ont sans doute un lien étroit avec le vieillissement du système de conduction cardiaque. En tout, $10 \%$ à $18 \%$ de la population totale des plus de 80 ans a été diagnostiqué de FA [1]. Ce nombre considérable de patients atteints de FA, estimé à environ 1 million de patients en France, continue d'augmenter à mesure que la population vieillit [2].

Quand on évoque la FA, on a tendance à penser d'abord à l'arythmie et au traitement pour la ralentir ou la réduire. Or, nous devrions penser peut-être d'abord à "embolie". L'embolie artérielle, le plus souvent cérébrale, est une des conséquences les plus fréquentes, et la plus dévastatrice, de la FA.

Environ un tiers de tous les AVC ischémiques sont d'origine cardio-embolique $[3,4]$. De plus, sur les AVC initialement étiquetés "cryptogéniques" (environ 25\% du total d'AVCs), jusqu'à 16\% s'avèrent présenter des paroxysmes de FA peu ou pas symptomatiques, quand on cherche l'arythmie assez longtemps avec des dispositifs portables [5]. Le traitement du risque embolique est en conséquence un composant essentiel de la prise en charge de la FA.

La personne âgée a non seulement un risque plus important de développer une FA, mais aussi, une fois qu'elle a une FA, un risque plus élevé d'embolie et d'AVC, et ceci 
en proportion directe à l'âge [6]. De ce fait, il faudrait que virtuellement tous les patients de plus de 75-80 ans avec une FA soient anticoagulés, sauf en cas de risque hémorragique excessif. Telle est bien la recommandation des derniers guides de pratique clinique des sociétés savantes européennes et françaises [7, 8]. Pourtant, la presque totalité des études réalisées montrent que les patients âgés avec FA sont paradoxalement le sous-groupe le moins bien anticoagulé, dans des proportions variables mais importantes: 30 à $50 \%$ des patients âgés qui devraient être anticoagulés, ne le sont pas $[9,10,11]$.

Nous voulions savoir si nos pratiques habituelles de prescription comportaient aussi une sous-prescription d'anticoagulation chez les patients âgés en FA hospitalisés dans notre service, un service hospitalier universitaire de gériatrie spécialisée, à orientation cardiologique, où en théorie nous devrions nous rapprocher au maximum des recommandations actuelles de traitement de ces patients. Si une sous-prescription existait, nous voulions connaître ses causes et ses caractéristiques. Pour cela nous avons suivi le traitement de tous les patients consécutifs admis avec une FA dans notre service pendant 3 mois. 


\section{Patients et Méthodes}

Il s'agit d'une étude observationnelle transversale.

\section{Patients inclus}

Nous avons inclus tous les patients âgés de plus de 75 ans avec un diagnostic de FA hospitalisés pour n'importe quel motif dans le service de gériatrie aiguë à orientation cardiologique (28 lits) de I'hôpital Charles Foix, à Ivry-sur-Seine (partie du groupe hospitalier Pitié-Salpêtrière-Charles Foix). Le diagnostic de FA pouvait être ancien ou être posé à l'occasion de l'hospitalisation, être un des motifs de l'hospitalisation ou être accessoire. L'étude a duré 3 mois, d'Avril à Mai 2013.

\section{Données recueillies}

Pour chacun des patients un investigateur indépendant $(\mathrm{HY})$ a recueilli, à partir du dossier médical, les prescriptions de traitement et l'entretien avec le médecin en charge, les données suivantes: a) antécédents: présence d'une hypertension artérielle, diabète, maladie rénale chronique, accident vasculaire cérébral, Alzheimer ou autre démence, trouble cognitif, maladie cardiaque, insuffisance cardiaque et saignement grave; b) développement pendant l'hospitalisation d'un saignement sévère; c) score CHADS2 de risque d'AVC embolique [12] et score HAS-BLED de risque hémorragique sous anti-vitamine $\mathrm{K}[\mathbf{1 3}]$; d) tout traitement antithrombotique au long cours à l'entrée de l'hôpital, médicament anticoagulant ou antiplaquettaire, et tout traitement antithrombotique à la sortie de l'hôpital; e) en cas de 
modification du traitement antithrombotique entre l'entrée et la sortie de l'hôpital, les raisons qui ont amené le médecin en charge à faire cette modification ; et, enfin, f) si le patient sortait de l'hôpital sans un traitement anticoagulant efficace, les raisons du médecin en charge pour ne pas anticoaguler le patient, ainsi que l'existence d'une anémie ou de troubles de l'hémostase.

Les médecins en charge des patients restaient libres du choix du traitement de leurs patients en tout moment. Aucune intervention n'a été effectuée pour modifier leur comportement à ce sujet.

\section{Statistiques}

L’analyse statistique a été purement descriptive, avec le calcul de proportions pour les variables qualitatives et de la moyenne et écart type pour les variables quantitatives. Tous les calculs ont été effectués avec le logiciel STATA (version 13, StataCorp LP, Texas, EEUU) 


\section{Résultats}

Au cours des 3 mois d'étude, 34 patients présentant une FA ont été hospitalisés dans le service. L'âge moyen était de de 85 ans (range : 75 à 99 ans). La FA était déjà connue pour 28 des patients, et un nouveau diagnostique pour 6 patients. Les caractéristiques de l'ensemble des patients sont présentées dans le Tableau 1.

\section{Traitement anti-thrombotique à l'entrée à l'hôpital et modifications postérieures :}

Le Tableau 2 récapitule l'ensemble des traitements antithrombotiques de ces patients.

Concernant les patients dont la FA a été découverte à l'occasion de l'hospitalisation, aucun des 6 patients ne prenait un traitement anti-thrombotique à l'arrivée (ni anticoagulation, ni antiagrégant plaquettaire). Par contre, 4 des 6 patients sont sortis de l'hôpital avec un traitement anticoagulant efficace (les 4 sous anti-vitamine K). Les raisons invoqués par leurs médecins pour ne pas anticoaguler les 2 patients restants étaient : a) un risque hémorragique jugé trop important du fait de chutes répétées, pour l'un ; b) difficultés d'observance et de sécurité d'administration du fait d'une démence évoluée, pour l’autre.

Parmi les 28 patients dont la FA était déjà connue, à l'arrivé à l'hôpital 20 suivaient un traitement anticoagulant avec des anti-vitamine $\mathrm{K}$ (10 fluindione, 9 warfarine), un patient était anticoagulé avec un nouvel anticoagulant oral direct (dabigatran), 4 
prenaient de l'aspirine, et 4 ne suivaient aucun traitement antithrombotique. Chez ces 28 patients, le traitement n’a été modifié que chez un seul patient, son traitement par aspirine étant remplacé par une anticoagulation efficace par warfarine.

Les 7 autres patients qui présentaient une FA connue et n'étaient pas anticoagulés sont sortis de l'hôpital avec leur traitement d'arrivé : 3 restaient traités par aspirine seule (le risque embolique jugé faible chez un patient, le risque hémorragique jugé trop élevé sous anticoagulation chez les 2 autres) et 4 sans aucun antithrombotique (l'un en raison d'un risque hémorragique jugé trop important, les 3 autres en raison d'une démence évoluée).

Le tableau 3 présente le statut anticoagulant de sortie d'hospitalisation pour les patients antérieurement non anticoagulés, en fonction de l'ancienneté du diagnostic de la FA. II y a une nette tendance à davantage d'initiation de l'anticoagulation chez les patients avec une FA nouvellement découverte, comparés aux patients avec une FA ancienne qui sont arrivés sans anticoagulation (test exact de Fisher, $p=0,09$ ).

\section{Caractéristiques des patients non anticoagulés :}

Au total 9 patients (26\%) sont sortis de l'hôpital sans anticoagulation efficace. Les caractéristiques individuelles de ces patients sont présentées dans le Tableau 4. Parmi les patients non anticoagulés, le score CHADS2 était de 2,3 +/- 0,8 (moyenne 
+/- écart type) et le score HAS-BLED de 1,9 +/- 0,9. Seuls 2 de ces patients avaient un risque hémorragique un peu supérieur au risque embolique cérébral selon ces scores (HAS-BLED $=3$ pour un CHADS2 $=2$, dans les 2 cas). Un seul patient avait un score CHADS2 inférieur à 2. Il n'y avait pas de patient avec anémie sévère, ni insuffisance rénale terminale ou créatininémie $>200$ micromole/L, ni perturbation apparente de I’hémostase. Un seul de ces patients présentait une thrombopénie à $84 \mathrm{G} / \mathrm{L}$. 


\section{Discussion}

Même si cette étude a la faiblesse évidente du faible nombre de patients inclus, ce qui ne permet pas de faire des estimations suffisamment fiables sur une population plus large, elle a la vertu d'illustrer trois aspects fréquemment constatés dans la pratique clinique réelle avec des patients âgés atteints d'une FA : a) il existe toujours une proportion non négligeable de patients âgés qui ne reçoivent pas une anticoagulation efficace sans une raison clairement valable, et ceci même dans un milieu spécialisé ; b) il est plus difficile de modifier les décisions sur le traitement anticoagulant prises par les médecins qui ont vu initialement le patient, que prendre une décision optimale chez un patient sans traitement qui vient d'être diagnostiqué de son FA ; et c) finalement, valables ou pas, la présence d'une démence évoluée et la crainte du risque hémorragique sont les motifs qui amènent le plus souvent à ne pas anticoaguler les patients âgés.

Cette étude a été menée dans un service hospitalier universitaire de gériatrie spécialisé, à forte orientation cardiologique. Les médecins du service connaissent très bien la FA, traitent couramment des patients âgés avec cette arythmie, sont au courant des recommandations les plus récentes, manient au quotidien les traitements anticoagulants et sont familiarisés avec les outils d'estimation du risque embolique (CHADS2, CHA2DS2-VASc) et hémorragique (HAS-BLED, HAEMORR2AGES) chez les patients en FA. Ce n'est pas, en conséquence, par manque de motivation, manque de connaissances ou manque d'expérience, qu'ils n'anticoaguleraient pas les 
patients âgés en FA. Pourtant, il y a une fraction significative ( $26 \%$ dans cette série) de patients en FA qui passent dans le service et qui sortent sans une anticoagulation efficace mise en route, sans raison valable apparente.

La présence d'une sous-prescription des anticoagulants chez des patients âgés n'a, en soi-même, rien de surprenant. Elle apparaît dans virtuellement toutes les études réalisées, en général dans des proportions plus élevées que dans cette série : entre $33 \%$ et jusqu'à $50 \%$ des patients $[9,10,11]$. Nous nous attendions, nonobstant - il faut l'avouer - à faire mieux (d'où l'intérêt d'évaluer nos pratiques). En tout cas, outre le constat de ne pas être dans l'idéal, ce qui est important est d'analyser le type de patients qui ne sont pas anticoagulés et les raisons qui le sous-tendent.

A l'examen des caractéristiques des patients ici non anticoagulés et de leurs scores CHADS2 et HAS-BLED, il n'apparaît pas, pour la plupart, une raison indiscutable pour ne pas anticoaguler. Néanmoins, les deux raisons les plus fréquentes données par les médecins qui ont pris cette décision ont été l'existence d'une démence évoluée, dans presque la moitié des cas, et un risque hémorragique jugé excessif, dans l'autre moitié. Comment expliquer cette discordance? II est possible qu'elle ne soit qu'apparente.

La présence d'une maladie démentielle évoluée a deux versants par rapport à une possible anticoagulation. D'une part, il y a le problème de l'observance et le suivi du traitement, qui peut être résolu avec l'intervention d'une tierce personne, aidant ou infirmière, pour s'occuper du traitement. D'autre part, et plus difficile à résoudre, il y 
a le questionnement sur le bénéfice apporté par un traitement comportant des contraintes et des risques certains à un patient qui a une expectative de vie limitée autant en durée qu'en qualité du fait de sa maladie neurodégénérative. Ceci peut être une raison légitime pour ne pas anticoaguler et elle ne sera recueilli par aucun score.

Concernant le risque hémorragique, l'appréciation par médecins des patients à forte risque dans cette série n'était pas confirmée par le score HAS-BLED pour la plupart des patients. Mais il ne faut pas oublier que les scores de risque hémorragique actuellement utilisés ont une fiabilité assez modeste, avec des statistiques " c " aux alentours de 0,65 (0,50 étant une classification entièrement au hasard et 1,00 étant une classification parfaite des patients) $[\mathbf{1 3}, \mathbf{1 4}]$. Surtout, ces scores ont été développés et validés dans la population générale et non chez le sous-groupe des patients âgés, pour lequel il n'existe actuellement aucun score de risque hémorragique spécifique, alors qu'il est connu que leur risque d'hémorragie est différent et plus élevé $[6,15]$. Un risque élevé de chute, par exemple, très fréquent chez les patients âgés, n'est tenu en compte que par un seul score, HAEMORR2AGES.

Finalement, Il est très intéressant de remarquer dans cette série que parmi les cas (7 patients sur 9) où un traitement anticoagulant n'avait pas été prescrit à la sortie de I'hôpital, la plupart étaient des patients dont la FA était connue et qui n'avaient déjà aucun traitement anticoagulant à leur arrivée. Par contre, chez la plupart des patients (5 sur 6) dont la FA a été découverte à l'occasion de leur hospitalisation, une 
anticoagulation a été prescrite ou une contre-indication formelle existait (1 patient avec risque hémorragique élevé). Ceci met en relief la difficulté à modifier les décisions de traitement prises par les médecins qui ont vu le patient avant nous, un biais cognitif bien connu en médecine, entre autres, sous le nom de biais $d^{\prime}$ « ancrage » $[16]$.

Outre la limitation évidente du faible nombre de patients inclus, ce qui ne permet pas de considérer les chiffres obtenues comme vraiment représentatifs d'une population plus large, les patients hospitalisés dans ce service ne sont pas forcément représentatifs de la population général des patients âgés avec FA. De plus le recueil des raisons indiquées par les médecins pour leur choix de traitement peut ne pas avoir tenu compte de l'ensemble des raisons (ex. raisons multiples). Malgré ces limitations, cette étude offre un aperçu plus détaillé que dans d'autres études des caractéristiques des patients âgés non anticoagulés et des raisons des médecins derrière cette décision.

\section{Conclusion}

Il persiste une proportion non négligeable de patients âgés avec FA sans traitement anticoagulant efficace, y compris dans un service hospitalier spécialisé. La présence d'une démence évoluée et la crainte du risque hémorragique chez le patient âgé semblent motiver une proportion importante de ces décisions de ne pas anticoaguler.

Le développement et validation d'outils d'évaluation du risque hémorragique 
spécifiques pour la personne âgé, permettant de faire des estimations plus fiables dans cette population, pourrait contribuer de façon importante à améliorer les décisions de mise sous anticoagulation dans cette population. 


\section{Références}

1. Heeringa J, van der Kuip DA, Hofman A, Kors JA, van Herpen G, et al. Prevalence, incidence and lifetime risk of atrial fibrillation: the Rotterdam study. Eur Heart J. 2006 Apr; 27(8): 949-53

2. Charlemagne A, Blacher J, Cohen A, Collet JP, Diévart F, et al. Epidemiology of atrial fibrillation in France: extrapolation of international epidemiological data to France and analysis of French hospitalization data. Arch Cardiovasc Dis. 2011 Feb; 104(2): $115-24$

3. Kolominsky-Rabas PL, Weber M, Gefeller O, Neundoerfer B, Heuschmann PU. Epidemiology of ischemic stroke subtypes according to TOAST criteria: incidence, recurrence, and long-term survival in ischemic stroke subtypes: a population-based study. Stroke. 2001 Dec 1; 32(12):2735-40

4. Marnane M, Duggan CA, Sheehan OC, Merwick A, Hannon N, Curtin D, et al. Stroke subtype classification to mechanism-specific and undetermined categories by TOAST, A-S-C-O, and causative classification system: direct comparison in the North Dublin population stroke study.. Stroke. 2010 Aug;41(8):1579-86

5. EMBRACE Investigators and Coordinators, Gladstone DJ, Spring M, Dorian P, et al. Atrial fibrillation in patients with cryptogenic stroke. N Engl J Med. 2014 Jun 26; 370(26): $2467-77$

6. van Walraven C, Hart RG, Connolly S, Austin PC, Mant J, Hobbs FD, et al. Effect of age on stroke prevention therapy in patients with atrial fibrillation: the atrial fibrillation investigators. Stroke. 2009 Apr ;40(4): 1410-6

7. European Heart Rhythm Association, European Association for Cardio-Thoracic Surgery, Camm AJ, Kirchhof P, Lip GY, Schotten U, Savelieva I, Ernst S, et al. Guidelines for the management of atrial fibrillation: the Task Force for the Management of 
Atrial Fibrillation of the European Society of Cardiology (ESC). Eur Heart J. 2010 Oct;31(19):2369-429

8. Hanon O, Assayag P, Belmin J, Collet JP, Emeriau JP, Fauchier L, et al; French Society of Geriatrics and Gerontology; French Society of Cardiology. Expert consensus of the French Society of Geriatrics and Gerontology and the French Society of Cardiology on the management of atrial fibrillation in elderly people. Arch Cardiovasc Dis. 2013 May;106(5):303-23

9. Nieuwlaat R, Capucci A, Lip GHY, Olsson SB, Prins MH, et al. Antithrombotic treatment in real-life atrial fibrillation patients: a report from the Euro Heart Survey on Atrial Fibrillation. Eur Heart J December 2006;27: 3018-3026

10. Gamra H, Murin J, Chiang CE, Naditch-Brûlé L, Brette S, Steg PG; RealiseAF investigators. Use of antithrombotics in atrial fibrillation in Africa, Europe, Asia and South America: insights from the International RealiseAF Survey. Arch Cardiovasc Dis. 2014 Feb;107(2):77-87

11. Lip GY, Laroche C, Dan GA, Santini M, Kalarus Z, Rasmussen LH, et al. 'Real-world' antithrombotic treatment in atrial fibrillation: The EORP-AF pilot survey. Am J Med. 2014 Jun;127(6):519-29

12. Gage BF, Waterman AD, Shannon W, Boechler M, Rich MW, Radford MJ. Validation of clinical classification schemes for predicting stroke: results from the National Registry of Atrial Fibrillation. JAMA. 2001 Jun 13;285(22):2864-70

13. Lip GY, Frison L, Halperin JL, Lane DA. Comparative validation of a novel risk score for predicting bleeding risk in anticoagulated patients with atrial fibrillation: the HAS-BLED score. J Am Coll Cardiol. 2011 Jan 11;57(2):173-80

14. Apostolakis S1, Lane DA, Guo Y, Buller H, Lip GY. Performance of the HEMORR(2)HAGES, ATRIA, and HAS-BLED bleeding risk-prediction scores in patients with atrial fibrillation undergoing anticoagulation: the AMADEUS (evaluating the use 
of SR34006 compared to warfarin or acenocoumarol in patients with atrial fibrillation) study. J Am Coll Cardiol. 2012 Aug 28;60(9):861-7

15. Hylek EM, Evans-Molina C, Shea C, Henault LE, Regan S. Major hemorrhage and tolerability of warfarin in the first year of therapy among elderly patients with atrial fibrillation. Circulation. 2007 May 29;115(21):2689-96

16. Croskerry, P. From Mindless to Mindful Practice - Cognitive Bias and Clinical Decision Making. N Engl J Med 2013; 368(26): 2445-8 


\section{Tableau 1. Caractéristiques des patients inclus}

Tous les valeurs, sauf quand spécifié autrement, en tant que : nombre (\%)

$\mathrm{n}=34$

\begin{tabular}{ll}
\hline Âge, moyenne (SD, range) & $85.4(6.02,75$ à 99) \\
\hline Femmes & $16(47)$ \\
\hline FA déjà connue & $28(82)$ \\
\hline FA découverte & $6(18)$ \\
\hline Antécédents & $24(71)$ \\
\hline HTA & $10(29)$ \\
\hline Maladie d'Alzheimer & $6(18)$ \\
\hline AVC & $5(15)$ \\
\hline Insuffisance cardiaque & $5(15)$ \\
\hline Insuffisance rénale chronique & $3(9)$ \\
\hline Diabète & $2,3(0,9)$ \\
\hline Scores & $1,8(0,8)$ \\
\hline CHADS2, moyenne (SD)
\end{tabular}

HTA : hypertension artérielle ; AVC : accident vasculaire cérébral 
Tableau 2. Traitements antithrombotiques à l'entrée et à la sortie de l'hôpital

\begin{tabular}{lll}
\hline & A l'entrée & A la sortie \\
& $\mathbf{n}$ & $\mathbf{n}$ \\
\hline FA déjà connue $(\mathrm{n}=28)$ & & \\
\hline Anti-vitamine $\mathrm{K}$ & $\mathbf{1 9}$ & $\mathbf{2 0}$ \\
\hline Fluindione & 10 & 10 \\
\hline Warfarine & $\mathbf{9}$ & $\mathbf{1 0}$ \\
\hline Nouveaux anticoagulants oraux & 1 & 1 \\
\hline Aspirine & $\mathbf{4}$ & $\mathbf{3}$ \\
\hline Aucun antithrombotique & 4 & 4 \\
\hline FA découverte $(\mathrm{n}=6)$ & & \\
\hline Anti-vitamine $\mathrm{K}$ & $\mathbf{0}$ & $\mathbf{4}$ \\
\hline Fluindione & $\mathbf{0}$ & $\mathbf{1}$ \\
\hline Warfarine & $\mathbf{0}$ & $\mathbf{3}$ \\
\hline Aspirine & 0 & 0 \\
\hline Aucun antithrombotique & $\mathbf{6}$ & $\mathbf{2}$ \\
\hline
\end{tabular}


Tableau 3. Traitement anticoagulant à la sortie chez les patients non anticoagulés à l'entrée de l'hôpital.

\begin{tabular}{lccc}
\hline & $\begin{array}{c}\text { FA de découverte } \\
\text { récente }\end{array}$ & FA ancienne & Total \\
\hline $\begin{array}{l}\text { Anticoagulation à la } \\
\text { sortie }\end{array}$ & 4 & 1 & 5 \\
Pas d'anticoagulation & 2 & 7 & 9 \\
Total & 6 & 8 & 14 \\
\hline
\end{tabular}

Il apparaît une tendance à davantage d'initiation de l'anticoagulation chez les patients avec une FA de découverte récente (test exact de Fisher, $p=0,09$ ) 
Tableau 4. Caractéristiques individuelles des patients sortis de l'hôpital sans anticoagulation efficace

\begin{tabular}{|ccccccc|}
\hline $\begin{array}{c}\text { Traitement } \\
\text { anti-thrombotique }\end{array}$ & $\begin{array}{c}\text { Score } \\
\text { CHADS2 }\end{array}$ & $\begin{array}{c}\text { Score } \\
\text { HAS-BLED }\end{array}$ & $\begin{array}{c}\text { Créatinine } \\
(\mu \mathrm{mol} / \mathrm{L})\end{array}$ & $\begin{array}{c}\text { Hémoglobine } \\
(\mathrm{g} / \mathrm{d} \mathrm{l})\end{array}$ & $\begin{array}{c}\text { Plaquettes } \\
(\times 10 * 9 / 1)\end{array}$ & $\begin{array}{c}\text { TP } \\
(\%)\end{array}$ \\
\hline Aspirine & 3 & 1 & 96 & 12,4 & 289 & 97 \\
\hline Aspirine & 4 & 3 & 63 & 11,9 & 242 & 109 \\
\hline Aspirine & 3 & 2 & 112 & 12,6 & 287 & 93 \\
\hline Aucun & 1 & 1 & 136 & 9,8 & 374 & 86 \\
\hline Aucun & 2 & 1 & 66 & 9,7 & 84 & 100 \\
\hline Aucun & 2 & 3 & 176 & 11,8 & 308 & 103 \\
\hline Aucun & 2 & 1 & 136 & 10,9 & 263 & 103 \\
\hline Aucun & 2 & 2 & 119 & 11,5 & 258 & 92 \\
\hline Aucun & 2 & 3 & 48 & 14 & 214 & 90 \\
\hline $\begin{array}{c}\text { Moyenne } \\
\text { (écart type) }\end{array}$ & $2,3(0,86)$ & $1,9(0,93)$ & $105(42)$ & $11,6(1,4)$ & $257(79)$ & $97(7,4)$ \\
\hline
\end{tabular}

